\title{
Conflictos entre culturas... verdad y testimonio
}

\section{Resumen:}

Los miembros de comunidades indígenas vencidas militarmente a fines del siglo XIXy principios del XX han recorrido, en la Argentina, un lento y tortuoso proceso para lograr ser tratados como ciudadanos en pie de igualdad frente a la ley. En el presente trabajo, se analiza este proceso desde la perspectiva de testimonios brindados por indígenas frente a la justicia, cuando estos fueron requeridos y valorados, aun cuando fueran contrarios a los de otras personas de la sociedad con mayor poder relativo. Se realiza el estudio de dos casos que afectaron a miembros de la etnia pilagá.

Palabras Clave: Multiculturalidad, Conflictos, Justicia.

\section{Summary:}

The members of indigenous communities that were militarily defeated at the end of the 19th and the beginning of the 20 th century have gone through a slow and tortuous process in Argentina to be treated as citizens on an equal footing before the law. In this work, this process is analyzed from the perspective of the testimonies of indigenous people to justice, when these were required and valued, even when they were contrary to those of other people in the society with greater relative power. The study of two cases that affected members of the pilagá ethnic group is carried out.

Keywords: Multiculturalism, Conflicts, Justice.

Mag. Diego Fernando Guichón / dieguichon@gmail.com/ https://orcid.org/0000-0002-54474960. El autor es Licenciado en Economía de la Universidad Nacional de Mar del Plata y Magister en Metodología de la Investigación Científica de la Universidad Nacional de Lanús. Recibido, 20/08/19, Publicado 24/05/20

Fuente de la foto: https://noticiasancap.files.wordpress.com/2015/10/pilagas2.jpg 
La pregunta que guía este trabajo es la siguiente: ¿cómo se han resuelto y se resuelven los conflictos interétnicos en dos circunstancias distintas, cuando impera la fuerza y cuando los conflictos se canalizan a través de un Poder Judicial, en un marco de igualdad al menos formal ante la ley? Nos interesa esta problemática en relación a las comunidades indígenas en Argentina.

Foucault (2007) ha buscado en la historia de las prácticas sociales confrontativas, particularmente en el ámbito jurídico, el lugar en que ha emergido el interés por la verdad y los primeros métodos para su obtención. Para este autor, además, ambas cuestiones se extenderán luego a las practicas científicas. El filósofo francés plantea un contraste notable entre los conflictos que se resolvían entre las partes y aquellos en que intervenía un tercero que poseían intereses propios en el conflicto y poder suficiente para resolverlo.

En el primer caso, la verdad y las evidencias que podrían haber proporcionado los testimonios poco importaban, y los conflictos se resolvían por mecanismos en los que primaba el poder, la fuerza y la habilidad en el marco de determinados procedimientos. En el segundo caso, hay un tercero con intereses propios distintos de los intereses de las partes y con poder para imponer su ley y controlar si la misma ha sido cumplida o no. En este contexto, emergen, de acuerdo a Foucault, la búsqueda de la verdad y, simultáneamente, métodos para hallarla, en particular el que denomina "indagación", que consiste en la recolección de testimonios sobre lo ocurrido. El autor encuentra rastros de esta modalidad en la tragedia "Edipo Rey", escrita por Sófocles en el siglo VA.C, en donde el testimonio de dos esclavos, que dicen aquello que han visto, permite condenar a un rey. Posteriormente, este interés por la verdad resurge en la Edad Media junto con la figura de un tercero, el Rey o Señor, que impone sus normas y actúa para velar por su cumplimiento a través de sus delegados, los procuradores. En este marco -señala Foucault- emerge, en la Europa Medieval, lo que luego se conocería como Poder Judicial. Esta historia, aunque propia de Europa, puede servirnos como guía en nuestro trabajo. Cabe preguntarse en qué momento, para la resolución de conflictos interétnicos, se planteó la necesidad de dilucidar la verdad por parte de un Poder Judicial dentro de un Estado-Nación. $\mathrm{Y}$ cuándo fueron buscados, escuchados y valorados los testimonios de personas con distinto poder en diferentes tiempos y lugares: los testimonios de los miembros de pueblos conquistados acerca de de miembros del pueblo conquistador, en otras palabras, los testimonios de miembros de etnias distintas. Como veremos, ambos momentos no son necesariamente simultáneos.

\section{Conflictos en la frontera}

Mientras la Argentina no se consolidó como Estado-Nación, es decir, mientras aún no tenía control completo sobre su territorio, podían darse relaciones diferentes: una con las comunidades indígenas que habitaban territorios no controlados por los gobiernos criollos, y otra con aquellas que estaban dentro de territorios controlados.

En el primer caso, las relaciones se daban particularmente en la zona de frontera y oscilaba entre momentos de enfrentamientos y momentos de acuerdos o tratados. Los conflictos que intentaban resolverse eran los que se derivaban del comercio interétnico, los cotos de caza, la entrega de cautivos, la vigilancia de fronteras e, incluso, las alianzas militares circunstanciales. No obstante, 
según Delrio (2002) y Bechis (2006), en el proceso de su conformación, también se buscó que las comunidades indígenas adhirieran al Estado-Nación, particularmente las comunidades de la zona de la Patagonia.

En este marco, lo que aparecía como subyacente era la ley del más fuerte ${ }^{1}$, dado que estos acuerdos o tratados se rompían cuando la relación de fuerzas cambiaba. Como señala Bechis (2006), el cacique Calfulcurá mantuvo un conjunto de acuerdos con el entonces Gobernador de la Provincia de Buenos Aires, Juan Manuel de Rosas, que se rompieron luego de la derrota de este en la batalla de Caseros. Esto dio lugar a diversos enfrentamientos, entre ellos un ataque indígena devastador, perpetuado hacia la población de Azul en 1855. Otros casos en los cuales los tratados se convirtieron en letra muerta frente al cambio en la relación de fuerzas fueron la denominada Masacre de Pozo del Cuadril ocurrida en 1878 (Delrio, 2010) y la rendición del cacique Sayhueque ante el Gobierno Nacional sobre el final de la denominada Campaña del Desierto (Delrio, 2002; Bechis, 2006;).

Una pregunta interesante a formularse frente a estos acuerdos es cómo se trató en esta instancia la diferencia de lenguas y el carácter ágrafo de la lengua indígena. Delos antecedentes consultados, se observa que los tratados se escribieron en castellano y que los líderes indígenas que los firmaron, salvo excepciones como Sayhueque, no podían escribirlos ni leerlos. Este punto ha sido

1El cambio en la relación de fuerzas en la frontera se relacionó con el propio proceso político y económico de construcción de territorio, población y mercado, que acompañó a la formación del Estado-Nación. Para ampliar este tema, ver también Delrio (2002) en referencia al cambio en el discurso oficial antes de la Campaña del Desierto, cuando se pasó de hablar de "Indios Amigos" a utilizar la expresión "Indios Salvajes". tratado por Bechis (2006), quien señala que cautivos y refugiados tenían un rol importante en la redacción de estos tratados. Los mismos eran sometidos a interrogatorios cruzados en distintas ocasiones para verificar la consistencia en las lecturas que se realizaban de aquello que se había escrito.

En esta etapa, mientras prevalecía el interés en mantener los tratados, es posible que hayan existido condiciones para dirimir los conflictos a través de la búsqueda de la verdad y de testimonios. Pero cuando el equilibrio de fuerzas se rompía, los tratados también, y los conflictos se resolvían por la ley del más fuerte. No existía un tercero por encima de las partes en conflicto que actuara como garante de cumplimiento de los acuerdos. No existía tampoco, necesidad de dilucidar la verdad, ni de testimonios para llegar a la misma.

\section{Conflictos al interior del Estado Nación}

Ahora bien, ¿qué ocurrió cuando las comunidades indígenas fueron vencidas militarmente a fines del siglo XIX en la Patagonia y a principios del siglo XX en el Chaco? En principio, como veremos, sus miembros no fueron tratados inmediatamente como ciudadanos de la Argentina, en pie de igualdad con otros ciudadanos. Por el contrario, esto se realizó luego de un proceso lento y tortuoso. En este trabajo, se analiza este proceso desde la perspectiva de los indígenas a partir de sus testimonios frente a la justicia, cuando los mismos fueron requeridos y valorados, aun cuando hayan resultado contrarios a los de otras personas de la sociedad con mayor poder relativo.

La investigación se basa en el estudio de dos casos que afectaron a miembros de la etnia pilagá. El primero de ellos corresponde 
al año 1899, y el segundo se desarrolla entre los años 1947 a 2010.

Consideramos que los dos casos de estudio son significativos, debido a su gravedad, derivada de los derechos vulnerados y de la cantidad de individuos afectados. Como los casos analizados son muy relevantes, la forma en que se han tratado puede brindar indicios sobre lo que ha ocurrido con casos de menor significatividad.

Nos interesa destacar en quémomento y en qué lugar se comenzó a buscar la verdad en los tribunales y, dentro de este proceso, se empezó a tomar en cuenta el testimonio de indígenas, aunque contradijera a personas social o políticamente importantes dentro de la Argentina. En otras palabras, en qué momento y lugar se alcanzó aquella situación que mencionaba Foucault que se logró ya en la Grecia de Sófocles 2400 años atrás, cuando el testimonio de dos esclavos permitió condenar a un rey. Además, para cada caso, se provee un análisis y su interpretación.

\section{Un contrato de locación de servicios del año $1900^{2}$}

En el año 1899, la etnia pilagá fue objeto de atención a nivel nacional. El famoso empresario teatral José Podestá celebró un contrato de locación de servicios, por el cual catorce mujeres de esta etnia, muchas menores de edad, serían trasladadas para ser exhibidas en un espectáculo en la Feria Internacional de Paris del año $1900^{3}$.

2 Todas las referencias de este caso son tomadas del historiador del derecho Abelardo Levaggi (1990, 1992).

3 La exhibición en circos y exposiciones de personas de pueblos colonizados forma parte de lo que se han denominado "zoológicos humanos", que fueron frecuentes hasta la década del treinta en Europa, como un ele-
El Defensor de Menores e Incapaces del entonces Territorio Nacional de Formosa rubricó este contrato en representación de estas mujeres y las mismas fueron trasladadas para ser embarcadas en Buenos Aires.

Cuando este hecho tomó estado público en Buenos Aires, se generó un escándalo que se reflejó en la prensa. A raíz del mismo, el entonces Ministro de Justicia e Instrucción Pública ordenó que las indígenas fueran alojadas en el Asilo del Buen Pastor, a partir de lo cual se entabló una batalla judicial. José Podestá entendió que las indígenas habían sido detenidas e interpuso un habeas corpus, al cual un juez federal de primera instancia hizo lugar. Este juez interpretó que el contrato era válido por haber sido firmado por el Defensor de Menores e Incapaces y entendió que el traslado de las indígenas a la Capital Federal constituía un principio de ejecución del contrato.

La sentencia fue apelada por el entonces Procurador de la Nación y la Corte Suprema de Justicia la revocó. El argumento del voto mayoritario de la Corte fue que las indígenas no se encontraban detenidas, sino en una condición en que podían manifestar libremente su voluntad. En el ínterin, un juez de Formosa, habiendo tomado conocimiento de los hechos, le solicitó al entonces Ministro de Justicia e Instrucción Pública que se tomara el testimonio de las propias indígenas.

De acuerdo a los antecedentes periodísticos relevados por Levaggi;

[...] ellas respondieron que habían sido embarcadas con engańo y en ausencia de sus esposos y padres, que ninguna manifestó el deseo de separarse deellos, yquese lamentaban

mento más dentro del contexto colonialista y racista de la época. El precursor de estas prácticas fue el alemán Carl Hagenbeck (1844-1913). 
deestar lejos. Días después fueron restituidas al ingenio en el que trabajaban y del que habían sido sacadas (Levaggi, 1990, p. 458).

\section{Análisis del caso}

A continuación, vamos a aportar algunos elementos que permitan comprender mejor este caso, en particular, por qué un tercero, un defensor de menores e incapaces, intervino en el contrato.

La Constitución Nacional de 1853 había atribuido al Congreso de la Nación la misión de proveer a la seguridad de las fronteras, conservar el trato pacífico con los indios y promover la conversión de ellos al catolicismo. En este texto, el indígena no era considerado como un ciudadano más, sino como parte de un grupo con el cual había que mantener la paz y asimilar culturalmente, al menos en materia religiosa. El status de ciudadanía del indígena resulta poco claro ${ }^{4}$.

En la Constitución de 1949, se eliminó el trato diferenciado hacialos indígenas; mientras que, en la Reforma Constitucional de 1994, se observa su reconocimiento como culturas particulares, que forman parte de un Estado, que poseen derechos propios y que, lejos de tener que ser eliminadas o asimiladas, deben poder preservar su identidad. Es en esta última reforma, cuando se abandona la idea del crisol de razas y se avanza con la posibilidad de un Estado multicultural. Existió, entonces, un largo período de tiempo, en el cual según la Constitución Nacional el status de las comunidades indígenas era o bien el de un extraño (hasta 1949), o bien el de alguien sin una identidad propia (entre 1949 a 1994).

En materia de derechos laborales, el destino de muchos indígenas luego de su

4 Ver Lenton (1999) sobre los debates parlamentarios en torno al tipo de ciudadanía que correspondía asignar al indígena. derrota militar fue el trabajo forzado o servil en distintos establecimientos agropecuarios, o como servicio doméstico en casas de familias acomodad ${ }_{\text {as }}^{5}$. Ahora bien, ¿cómo explicar esta situación si formalmente la abolición de la esclavitud en la Argentina fue un proceso que se inició con la Asamblea del Año $1813^{6}$ y culminó con la Constitución de 1853?.

Otro antecedente a considerar tuvo lugar cuando, en 1921, Hipólito Irigoyen envió al Congreso de la Nación un proyecto de Código de Trabajo que proponía que no hubiera ninguna diferencia entre los trabajos del indígena y del resto de los obreros (Martínez Sarasola, 1998, pp. 194-195). No obstante, este proyecto no prosperó. Con lo cual la pregunta que puede hacerse es: si no había igualdad ante la ley, ¿cuáles eran los derechos que amparaban a los trabajadores indígenas? Nuevamente nos encontramos con un interrogante.

Levaggi brinda algunos elementos para dilucidar esta situación. El autor (Levaggi, 1990, 1992) sostiene que, luego de las declaraciones de igualdad frente a la ley, que comenzaron ya en 1811 , se produjo en forma paulatina una vuelta al derecho indígena de la Colonia, en donde se les reconocía a los indígenas un estatus jurídico inferior y se encomendaba su tutela a los defensores de naturales, quienes fueron

5 Sobre la distribución de indígenas y los procesos típicos de asimilación con la consiguiente pérdida de identidad, ver el trabajo de Escolar (2008).

6 En la Asamblea del Año 1813, se denomina a los indígenas: "Hombres perfectamente libres, en igualdad de derechos con todos los demás ciudadanos que pueblan las provincias". El artículo 128 de la Constitución de 1819 se refirió a los indígenas como "iguales en derechos y en dignidad a los demás ciudadanos, regidos por las mismas leyes y llamados al goce de las mismas preeminencias" (citado en Levaggi, 1990, pp. 446-450). 
reemplazados de hecho por defensores de menores o incapaces ${ }^{7}$.

Dentro de los antecedentes a nivel nacional citados por este autor, resulta de particular interés el Decreto No 11.316 del 22 de agosto de 1879, suscripto por el entonces Presidente Nicolás Avellaneda. En el artículo 1 se resuelve:

Queda a cargo del defensor nacional de pobres e incapaces la colocación de las familias y menores indígenas, prisioneros por las fuerzas nacionales, a cuyo efecto una vez trasladados a esta ciudad serán puestos a su disposición por el Ministerio de la Guerra (Citado en Levaggi, 1990, p. 454).

Posteriormente, el 3 de mayo de 1899 , durante el primer mandato presidencial de Julio Argentino Roca, se emitió un nuevo Decreto. Este ampliaba las competencias de los defensores de menores de los Territorios Nacionales, quienes debían actuar como:

\section{[...] defensores y protectores de los in-} dígenas en todo cuanto beneficie a éstos, debiendo proveer por cuenta del Estado a su alimentación, vestido y colocación, y ejercer respecto de ellos en todo lo demás su acción tutelar, mientras sea necesario (Citado en Levaggi, 1990, p. 455) ${ }^{8}$.

Por medio de estos decretos, se estableció ya a nivel nacional lo que se había instituido como una práctica en diversas provincias de la Argentina. Como si fuese una ficción jurídica, pueblos enteros y los miles de individuos que los componían fueron considerados jurídicamente incapaces. Las

7 En el derecho colonial, un concepto jurídico aplicado, equivalente al de "incapaz", fue el de "indio miserable". Sobre este tema, ver Cunill (2011).

8 Según pudo constatarse, los decretos de dicho año, publicados en el Registro Nacional de la República Argentina, en muchos casos, carecen de número. causas de esta incapacidad, sellamarán estas "incivilización", "rusticidad" o "ignorancia", no estaban previstas en el Código Civil de Vélez Sarsfield. No obstante, a partir de estos decretos, el representante de los indígenas, el Defensor de Menores e Incapaces, pudo actuar por su sola iniciativa y sin concurso de la voluntad del indígena "incapaz". No fue necesario ya escuchar lo que el indígena opinara para decidir sobre él y su familia.

Dentro del instituto de la incapacidad, estaban previstos dos tipos de tutela: por un lado, los tutores o curadores, que tenían a su cargo las acciones diarias y, por otro lado, las defensorías especializadas. Sin embargo, para toda esta multitud de indígenas "incapaces", no se designaron tutores ni curatos, sino que su representación y protección quedo a cargo exclusivamente de defensores oficiales.

Sin perjuicio de lo injusto del sistema, el mismo era totalmente ineficaz. Si se piensa en los lugares inicialmente remotos en que se asentaron muchas comunidades indígenas y en los medios de transporte y comunicación de ese entonces, se apreciarán las dificultades prácticas que implicaba este mecanismo de defensa y protección. Tampoco queda claro cómo estos defensores oficiales podrían proveer, por cuenta del Estado, alimentación y vestido.

Bialet Massé (1904) y Levaggi (1990) señalan el fracaso de este esquema que, de acuerdo a este último autor, fue una política común a todas las repúblicas hispanoamericanas. En tal sentido, en nuestro país, si bien se promovieron varias iniciativas para instituir, sea en forma privada o en forma pública, la figura del Patronato que se ocupase de los indígenas, ninguna de ellas llegó a concretarse.

Ahora bien, resulta claro que la combinación de incapacidad jurídica y de au- 
sencia de recursos para su representación y protección efectiva fue una síntesis nefasta de desamparo para el indígena, que facilitó la existencia de relaciones de servidumbre y de todo tipo de abuso en materia laboral.

En este contexto, si bien existía un Poder Judicial que debía buscar la verdad para resolver los conflictos, el testimonio de los indígenas no tenía ningún valor en dicho proceso. En efecto, fueron solo las consideraciones políticas que se introdujeron en el caso de las indígenas traídas desde Formosa lo que llevó a escuchar sus voces, dado que, desde un punto de vista jurídico, eran personas incapaces.

\section{El episodio de Rincón de la Bomba 1947-2010}

Analizaremos, en este apartado, un conflicto mucho más grave por sus consecuencias irreparables, el cual ha sido el último episodio conocido hasta la actualidad de matanza o masacre masiva de integrantes de una comunidad indígena. Este caso se desarrolló entre el momento en que ocurrieron los hechos en 1947 y el año 2010 cuando, como veremos, se tomó el testimonio de los indígenas en una forma especial.

En el año 1947, se llevó a cabo lo que se considera como la última masacre de indígenas en Argentina, en el paraje La Bomba, o Rincón de la Bomba, ubicado cerca de la localidad de Las Lomitas, en lo que, en ese tiempo, se denominaba Territorio Nacional de Formosa. En el mes de octubre, y durante varios días, efectivos deGendarmería Nacional, con armas pesadas y el apoyo de un avión militar, asesinaron a un número indeterminado de indígenas pertenecientes a la etnia pilagá. El número de víctimas no ha sido determinado con certeza, pero, en el año 2013, las fuentes judicialesya reconocían másde 80 fallecidos ${ }^{9}$, mientras quelosindígenas

9 Ver el testimonio en video del Fiscal de la sobrevivientes y sus representantes llevaban ese número a centenares o miles. ${ }^{10}$

El episodio de Rincón de la Bomba estuvo precedido por otro hecho de violencia extrema, que también ha sido considerado una masacre de indígenas. Nos referimos al episodio de Napalpi en el Territorio Nacional del Chaco en 1924 (Andre, 2012, 28 de julio). En este caso, participaron también fuerzas de distintos niveles de gobierno. Ahora bien, el episodio de Napalpi tuvo como antecedente una huelga laboral y, en este sentido, guarda relación con otros episodios contemporáneos, tales como la Semana Trágica (1919), la Patagonia Trágica (1921) y la Forestal (1921), todos los cuales terminaron en masacres de trabajadores. ${ }^{11}$ El episodio de Rincón de la Bomba (1947) fue diferente, porque ocurrió durante un gobierno en el que ya no se reprimían las huelgas como en la década del veinte e, incluso, existían mayores derechos laborales, como los logrados a partir del Estatuto del Peón Rural, dictado en 1944.

Delostestimonios sobre el hecho, se infiere que en Rincón de la Bomba inicialmente se produjo una concentración con fines religiosos. Esta concentración llamó la atención de las autoridades locales y nacionales, quienes consideraron la situación peligrosa. Para dispersar a los participantes, se realizaron gestiones, en las cuales tomó parte personal dela Dirección de Protección del Aborigen. Posteriormente, se

causa, Dr. Horacio Rodríguez (Memorias de la Tierra, 2012, 1 de junio).

10 Los detalles de esta masacre pueden consultarse en varias publicaciones, entre las cuales citamos a Delrio et al (2011) y Lenton (2014).

11 No obstante, los testimonios de lo ocurrido en Napalpi muestran un tipo de represión aún más brutal, con mutilaciones y violaciones, que no podemos afirmar que haya ocurrido en los otros casos. 
desató la acción militar, que se materializó en la matanza. En informes oficiales yen diarios de laépoca, se mencionaba la existencia previa de un malón, que luego se corroboró que nunca había existido. No se ha identificado en este evento evidencia alguna de que se haya dado intervención a la justicia.

Luego del hecho, siguió un largo período sin que se registraran referencias de lo sucedido. En efecto, entre octubre de 1947 y el año 2005, no hemos encontrado antecedentes de que se hayan radicado denuncias, ni de investigaciones judiciales de oficio. Tampoco hemos encontrado textos académicos en donde se mencione la matanza. Más allá de los testimonios, que podían aportar evidencia de lo ocurrido, no habría actuado nadie en forma oficial o particular para dilucidar la verdad.

Matarrese (2017) expone que, hasta la década del setenta inclusive, el territorio que ocupaba la comunidad pilagá se vio afectado adversamente por el progreso agropecuario. A partir de 1984, bajo una nueva legislación nacional, las comunidades indígenas emplazadas en la Provincia de Formosa comenzaron un proceso para el reconocimiento de su personería jurídica $y$, posteriormente, lograron la entrega en propiedad de diversos territorios distribuidos en dicha provincia (Matarrese 2018). En este nuevo contexto, en el año 2005, la Federación Pilagá, entidad con personería jurídica, interpuso una demanda en los tribunales ordinarios por sus derechos violados en 1947.

Se argumentó que existió un crimen de lesa humanidad y que, por lo tanto, no tiene prescripción. Se iniciaron, entonces, investigaciones forenses que determinaron la existencia de veintisiete cadáveres, lo que se correspondía con los hechos denunciado. Por otra parte, se allanó el cuartel de Gendarmería de Las Lomitas.
Ahora bien, a pesar de lo reciente de los procesos judiciales, llegó a materializarse el testimonio de los indígenas sobrevivientes antes de que muchos de ellos fallecieran por causas naturales. No obstante, los testimonios no fueron recolectados por el impulso del poder judicial actuante, sino que se debió, inicialmente, a la acción que decidió emprender la documentalista Valeria Mapelman. Tal como señaló esta directora cinematográfica en un reportaje:

Durante cuatro años recorrimos las comunidades, nos encontramos con sobrevivientes, quienes manifestaron que estuvieron esperando todos estos años a que alguien viniera a preguntarles qué había sucedido.

Siempre dijeron que era la primera vez que alguien se sentaba realmente a escuchar lo que había pasado. Así que prendimos la cámara, filmamos sin parar y sin interrumpirlos porque ellos hablaban en pilagá. Después, tradujimos todo. Y en base a todo ese primer acercamiento, se armó el guion (Ranzani, 2010, 12 de abril).

Agrega en este sentido la documentalista:

Coincidimos con la búsqueda de pruebas que estaba realizando un forense, pero la propuesta del documental fue recopilar las memorias de los ancianos sobrevivientes porque se están muriendo. Son muy viejitos. Si se mueren, se acabó: no hay quién lo cuente, porque ellos no tienen libros ni la posibilidad de publicar lo que pasó. Entonces, había que ir y recopilar esas memorias de alguna manera (Ranzani, 2010, 12 de abril).

Tal como señala Mapelman, los pilagás expresan con claridad que "estuvieron esperando estos años a que alguien viniera”. En un contexto de silencio del indígena (Bengoa, 1995), ellos no concurrieron a la Justicia, pero sí esperaron la presencia de un tercero interesado en la verdad y que 
valore sus testimonios. En base al material reunido, en el año 2010, se presentó el documental Octubre pilagá. Relatos sobre el silencio (Mapelman y Varreire, 2010), en donde ya traducidos pudieron escucharse por primera vez los diversos testimonios de los sobrevivientes. Con este documental se rompió definitivamente el silencio de esta comunidad indígena y sus testimonios adquirieron valor como evidencia. Se ha alcanzado, sesenta años después del episodio, el umbral a partir del cual el testimonio de los indígenas será tomado en cuenta, aunque el mismo incrimine a autoridades nacionales de distinto nivel.

En el momento en que la causa se inició, muchos ciudadanos vinculados a la misma ya habían fallecido, mientras que otros fallecieron en los ańos posteriores, sin haberse visto afectados por este juicio. Tal es el caso del Dr. Leandro Santos Costas, quien, en su condición de alférez de gendarmería, estuvo a cargo de una de las ametralladoras con que se realizó la masacre de Rincón de la Bomba. Luego, gracias a sus estudios de derecho, fue miembro del Poder Judicial de Formosa e integrante de la Cámara Federal de Justicia de dicha provincia, de donde se retiró en 1999. Santos Costas falleció en el año 2012.

\section{Análisis del caso}

Este caso amerita una explicación histórica más compleja, que integre el análisis del primer caso como uno de sus momentos.

Nuestra hipótesis es que, desde la derrota militar de los indígenas hasta la actualidad, se pueden identificar condiciones históricas que permiten inferir cierta forma predominante de tratamiento de conflictos. Estas condiciones implican aspectos normativos, pero también prácticas administrativas y sociales, que no necesariamente surgen de las normas.
En lugar de condiciones, hablaremos de "contextos", los cuales tienen asociados modalidades predominantes de tratamiento de conflictos. Nos interesa destacar bajo cada contexto identificado si predomina o no la búsqueda de la verdad y de testimonios, en particular, de los indígenas involucrados.

Entendemos que este tipo de explicación implicasiempre una interpretación dela situación histórica, que podría ser objeto de estudios adicionales. En tal sentido, una descripción de un contexto es inevitablemente parcial y otros elementos podrían incorporarse. Por ejemplo, la revisión de normas provinciales y de la jurisprudencia en la materia. Por otra parte, se mencionan algunas prácticas sociales a partir de fuentes secundarias, pero también es una tarea que podría ampliarse.

\section{Marco normativo}

Un primer elemento que conviene analizar se refiere a los derechos formales de los indígenas una vez vencidos militarmente. Si no se toman en cuenta las diferencias culturales, hay al menos cuatro posibilidades que están íntimamente relacionadas con la cuestión de la ciudadanía, a saber:

a) Los indígenas no son considerados ciudadanos o tienen una ciudadanía parcial. En esta instancia, hay una discriminación negativa: se les niega derechos que otros ciudadanos poseen.

b) Alos indígenas se los considera en pie de igualdad con el resto de los ciudadanos. En esta etapa, no hay discriminación negativa hacia las comunidades indígenas en el sentido de negarles derechos que otros ciudadanos poseen.

c) Seles reconocen a las etnias originarias ciertos derechos especiales, tal como es el caso 
de la propiedad comunitaria, incluido en el derecho de USA en 1934 y en la reforma de la Constitución Argentina de 1994. Este sería un caso de discriminación positiva, en el cual las etnias indígenas tendrían derechos adicionales, fundamentados en su cultura, que no estarían disponibles para otros ciudadanos. ${ }^{1213}$

d) Se considera que hay Plurinacionalidad. En este último caso, se rompe el esquema del Estado-Nación, en donde todo se tiende a unificar: un único poder soberano, un único derecho, etc. En la Plurinacionalidad, hay diversidad de Derechos ${ }^{14} \mathrm{y}$

12 En relación a las prácticas de discriminación positiva hacia comunidades indígenas, cabe señalar que la Reforma Constitucional de 1994, en el inciso 17 de su artículo 75, dispone: el respeto a su identidad, el derecho a una educación bilingüe e intercultural, el reconocimiento a la personería jurídica de sus comunidades y la posesión y propiedad de las tierras que tradicionalmente ocupan, y su participación en la gestión referida a sus recursos naturales y a los demás intereses que los afectan. Una descripción más completa puede encontrarse en Congreso de la Nación Argentina (Dirección de Servicios Legislativos, 2018), en donde se recopilan también fallos de tribunales provinciales y nacionales vinculados a la Reforma Constitucional de 1994.

13 Un caso típico que da cuenta de esta nueva situación que está en pleno desarrollo son las pericias antropológicas en el ámbito de justicia penal que son requeridas generalmente por las defensas de indígenas para argumentar razones culturales para su inocencia. Sobre este punto, ver Carrasco (2016).

14 La jurisdicción indígena es algo que tiene un escaso desarrollo en la Argentina. Según Bialet Massé (1904), hay algunos casos muy particulares en donde la Corte Suprema de Justicia rechazó intervenir en cuestiones que consideró estaban regladas por el derecho y las costumbres indígenas, tales como el dere- se plantean cuestiones complejas de cómo pueden interactuar los mismos. ${ }^{15}$

Este enfoque formal, al omitir las diferencias culturales, puede dar lugar a una visión inadecuada. Por ejemplo, si se pudiesen identificar ciertos derechos humanos básicos, tales como el derecho a la vida, a la libertad, a la integridad física, a la libre circulación, como condiciones necesarias para otro tipo de derechos, tales como el derecho a la propiedad comunitaria, cabría preguntarse de qué vale el derecho a la propiedad comunitaria, si no está protegido el derecho a la vida.

No obstante, este enfoque omite que no necesariamente existe una misma jerarquía de valores entre distintas culturas. En el estudio de este caso, hemos podido advertir cómo solo cuando las comunidades pilagá tuvieron un reconocimiento efectivo de derechos sobre la tierra que ocupan, es decir, cuando pudieron evitar esa vulnerabilidad, fueron capaces de llevar adelante los reclamos por el derecho a la vida de sus paisanos. Como si el derecho a la vida no pudiese defenderse si no es a partir de un derecho a la tierra ya consolidado.

Por lo expuesto, en este trabajo, se considera que el marco normativo es insuficiente para explicar la forma de resolución de conflictos. A las diferencias culturales ya mencionadas, cabe agregar los vacíos o ambigüedades en la normativa, y las políticas y las prácticas administrativas y sociales que no se reflejan necesariamente en la misma.

cho sucesorio, como lonco o cacique.

15 Ver, sobre este último enfoque, los casos de Bolivia y Ecuador, analizados en de Souza Santos y Rodríguez (2012). 


\section{Contextos}

Por contextos, nos referimos a una descripción amplia, que no incluya solo el marco normativo, sino también prácticas políticas $\mathrm{y}$ sociales. A partir de las fuentes secundarias consultadas, consideramos que es pertinente hablar de tres contextos distintos:

a) Seguridad-Peligrosidad. Bajo este contexto no existen o se suspenden los derechos civiles de los indígenas. No están dadas las condiciones para que intervenga el Poder Judicial y sea posible una búsqueda de la verdad o de testimonios para la resolución de conflictos.

b) Silencio del Indígena. Bajo este contexto pueden o no existir formalmente derechos civiles, pero, aunque los haya, en la práctica los mismos no están garantizados. El indígena, que es consciente de esta situación, opta por el silencio y la justicia no lo interpela. Por razones diferentes a las del primer contexto, aquí tampoco están dadas las condiciones para la búsqueda de la verdad en la resolución de conflictos, ni la búsqueda de testimonios.

c) Trato Equitativo. Bajo este contexto el indígena o sus organizaciones se hacen escuchar en la justicia y utilizan los recursos que la misma provee. En estas condiciones, el rol del Poder Judicial, y del debido proceso, es importante para la resolución de conflictos.

Estos tres contextos no cambian de manera uniforme en todo el país, sino que, en determinadas regiones o provincias, evolucionan más lentamente que en otras. Tampoco se dan simultáneamente en todas las áreas de conflicto, por ejemplo, en materia de derechos de propiedad la evolución puede ser distinta que en materia del ejercicio de derechos laborales. Tampoco presentan una evolución lineal irreversible, dado que,

\section{Introducción}

eventualmente, por ejemplo, el contexto de Seguridad-Peligrosidad puede parecer ${ }^{16}$ cuando ya se lo suponía superado. Otra posibilidad es que el contexto de Silencio del Indígena retorne cuando ya había sido abandonado en favor de un Trato Equitativo.

En los dos primeros contextos, los conflictos se resuelven predominantemente por la vía de hecho. La diferencia es que, en el segundo contexto, debería intervenir un Poder Judicial para dilucidar la verdad, pero esto no ocurre. Los derechos de los indígenas no existen o son poco claros, no hay un tercero con interés efectivo en hacer cumplir los mismos, los indígenas no comprenden el régimen legal o desconfían de este. Solo bajo el tercer contexto intervendrán los tribunales, se buscará que exista el debido proceso, habrá interés en dilucidar la verdad de lo ocurrido y tendrá valor el testimonio de todos aquellos que tengan algo que decir sobre los hechos, independientemente del grupo étnico al que pertenezcan.

\section{El contexto de}

\section{"Seguridad-Peligrosidad"}

El ejemplo más directo que puede darse de este contexto es el que apareció inmediatamente después de la derrota militar de los indígenas. Los indígenas vencidos a fines del siglo XIX en las campañas militares emprendidas en La Pampa, en el norte de la Patagonia y en el Chaco eran, en muchos casos, recluidos en centros de detención, bajo jurisdicción militar. Los casos más conocidos -pero no los únicos- fueron los de la Isla Martin García, en el Rio de la Plata (Nagy y Papazian, 2011), y Valchetta, en la

16 Lenton (2010) ilustra esta situación con el golpe de Estado de 1976 y la doctrina de la Seguridad Nacional, que afecta a toda la población, pero de una forma particular a las comunidades indígenas. 
Provincia de Río Negro (Delrio et al, 2010; Pérez, 2015).

El indígena continuaba retenido un tiempo, no porque hubiera violado alguna ley y hubiera sido sentenciado por eso, sino porque era un prisionero que se consideraba virtualmente peligroso. Típicamente se suponía que aún podía agruparse y atacar a través de malones y ser así una amenaza para otras poblaciones ${ }^{17}$.

No hay mucha información sobre lo ocurrido durante dichas detenciones (Pigna, 2004), pero es evidente que no existieron derechos de ciudadanía.

El episodio de Rincón de la Bomba, en octubre de 1947, es, de acuerdo a nuestro enfoque, un hecho que ocurrió en un contexto donde priman razones de Seguridad-Peligrosidad y en el cual no existen o no se toman en cuenta los derechos de los indígenas.

\section{El contexto de \\ "Silencio del Indígena"}

La expresión silencio del indígena la tomamos de Bengoa (1995), quien señala que las revoluciones del siglo XIX en América Latina plantearon como uno de sus principios básicos el de igualdad frente a la ley, pero que el mismo no se aplicó con relación a la población indígena. En tal sentido, Mariategui (1928) se refiere a un concepto particular, acuñado en Perú y Ecuador, el "gamonalismo", que servirá para visualizar la situación en aquel momento. Según este autor:

El "gamonalismo" invalida inevitablemente toda ley u ordenanza de protección indígena. El hacendado, el latifundista, es un

17 Escapa al alcance de esta investigación determinar qué rol podían cumplir en estos casos los códigos militares. señor feudal. Contra su autoridad, sufragada por el ambiente y el hábito, es impotente la ley escrita. El trabajo gratuito está prohibido por la ley y, sin embargo, el trabajo gratuito, y aun el trabajo forzado, sobreviven en el latifundio. El juez, el subprefecto, el comisario, el maestro, el recaudador, están enfeudados a la gran propiedad. La ley no puede prevalecer contra los gamonales. El funcionario que se obstinase en imponerla sería abandonado y sacrificado por el poder central, cerca del cual son siempre omnipotentes las influencias del gamonalismo, que actúan directamente o a través del parlamento, por una y otra vía con la misma eficacia (Mariategui, 1928, pp. 19).

De acuerdo a Bengoa (1995), el "gamonalismo" corresponde al periodo del "silencio indígena". Nuevamente empleando fuentes secundarias, este trabajo considera que los episodios, que narra Borrero (1989), de violencia extrema contra los indígenas a manos de hacendados privados en la Patagonia a fines del siglo XIX y principios del siglo XX serían casos de "gamonalismo" local.

Veamos ahora algunos antecedentes que permiten visualizar mejor el caso analizado.

Respecto de la situación ambigua de los derechos indígenas, que deja vacíos y situaciones precarias, existen varios ejemplos ${ }^{18}$. Ya nos referimos, en el caso de las catorce mujeres de la etnia pilagá, al tratamiento dado a los indígenas en la Constitución, al régimen de incapacidad jurídica en que fueron encuadrados inicialmente y a las consecuencias en materia de derechos laborales.

Las políticas de reducción, protección y civilización fueron la continuación en el siglo XX de estas prácticas iniciadas en el siglo XIX. Estas políticas se comenzaron

18 Ver Lenton (1997) para un análisis de los cambios que ocurren en el tratamiento de la cuestión indígena en el Congreso de la Nación. 
a instrumentar inmediatamente después de la derrota militar a fines del siglo XIX. ${ }^{19}$ Martínez Sarasola (1998) expone algunos elementos de este proceso.

La política de reducción se orientó a mantener a las comunidades indígenas en un territorio determinado. La Ley 4.167 del año 1903 regulaba un régimen de tierras fiscales y establecía que "el Poder Ejecutivo fomentará la reducción de las tribus indígenas procurando su establecimiento por medio de misiones y suministrándole tierra y elementos de trabajo". No obstante, estas asignaciones de tierra fueron mayormente precarias y los indígenas estuvieron sujetos a ser relocalizados. Además, sus derechos de uso de la tierra también podían ser cercenados (Delrio, 2010; Fandos y Teruel, 2012).

Intervinieron, entonces, prácticas políticas y administrativas de asignación de tierras por las cuales las comunidades indígenas resultaron particularmente vulnerables. Para estas, el "progreso" constituyó una amenaza. En efecto, a medida que mejoraban las comunicaciones y la accesibilidad a los territorios que ocupaban, mayor riesgo existía de que los mismos fueran reclamados por terceros para el desarrollo de actividades económicas y de que las comunidades fueran relocalizadas. Por otra parte, el propio trabajo del indígena, en la medida en que introducía mejoras en el territorio que ocupaba, generaba un riesgo similar; mientras que si, por el contrario, no introducía mejoras, confirmaba el estereotipo de pueblo incivilizado ${ }^{20}$.

19 Según Martínez Sarasola (1998), hay episodios de resistencia armada indígena en el Gran Chaco hasta el año 1936. Recién a fines de 1938, el ejército puso fin a sus operaciones bélicas y de "limpieza". Sin embargo, las políticas hacia los indígenas vencidos comenzaron mucho antes.

20 Sobre este punto, cabe citar a Bialet Massé (1904, pp. 40) y el fallo de la Corte Suprema de la Nación del 21 de julio de
Las políticas de "protección" trataron de suplir, desde el Poder Ejecutivo, las insuficiencias de los defensores de menores e incapaces. En 1912, se estableció por Decreto que el trato con los indios quedaba a cargo de la Dirección General de Territorios Nacionales. En 1916, se creó una Comisión Honoraria de Reducciones de Indios, que comenzó a funcionar, de acuerdo a Lenton (1997), recién en 1927. En 1946, fue reemplazada por la Dirección de Protección del Aborigen, dependiente de la entonces Secretaría de Trabajo y Previsión.

Las políticas de instrucción o civilización promovieron la sustitución de la cultura propia de estas comunidades para que fueran incorporadas al mundo "civilizado". En la Ley 1.532 del año 1884, que organizó los Territorios Nacionales dentro del país, solo se mencionaba la política de asimilación y se encomendaba específicamente a los Gobernadores que los indígenas que habitaran en los territorios bajo su mando fueran traídos "gradualmente" a la civilización.

Otra práctica administrativa que estudia Lenton (1995) fue la entrega de documentos personales de identidad. En 1885, se abandonó el sistema de Registros Parroquiales y se creó el Registro Civil de la Ciudad de Buenos Aires y los Territorios Nacionales. Posteriormente, en 1911, a partir de la ley

1969 en los autos "López Abdon s/recurso de amparo" (Peró et al, 1969), en donde se le negó a un miembro de la tribu tehuelche de la reserva Camusu Aike, en la Provincia de Santa Cruz, el derecho a utilizar las tierras que ocupaba para el pastoreo de una cierta cantidad ovejas. En nuestra opinión, es notable por su claridad y justicia el voto en disidencia del Dr. Marco Aurelio Risolía, que lamentablemente no prevaleció y que evidencia cómo determinadas prácticas administrativas contribuyeron a mantener a los indígenas en la pobreza. Asimismo, es notable este voto por ser emitido en el contexto de una Dictadura Militar. 
8.129, se dispuso el enrolamiento de toda la población masculina mayor de 18 años. Esta función quedó a cargo de las autoridades militares, a las cuales se subordinaron para el cumplimiento de este objetivo, las oficinas del Registro Civil ${ }^{21}$. El Documento de Identidad pasó a denominarse Libreta de Enrolamiento de manera que el ejercicio de derechos políticos y el cumplimiento del servicio militar obligatorio quedaron directamente vinculados. De acuerdo a Lenton (1999), quien ha estudiado este proceso, fue solo recién a partir del año $1947^{22}$ cuando se comenzó a entregar sistemáticamente la documentación civil a los indígenas, lo que les permitió el ejercicio de varios derechos, entre ellos, el derecho a voto ${ }^{23}$.

El desamparo del indígena queda en evidencia en algunos testimonios. Bialet Massé (1904) relata la existencia de contratos escritos entre comunidades indígenas y empresarios que no se cumplían. También expone cómo, en la medición de los pagos en especie, se adulteraban las cantidades 24 .

21 Todo esto resultaba compatible con la ausencia de derecho a voto de las mujeres.

22 Existieron acciones previas aisladas. En el año 1937, se crearon las primeras oficinas del registro civil en colonias indígenas (en Napalpi y Bartolomé de las Casas) para extender documentos de identidad.

23 De acuerdo a Lenton (1999), el proceso fue simultáneo con el reconocimiento del derecho a voto femenino. La Ley 13.010 habilitó el voto femenino y, posteriormente, la ley 13.482 creó el Registro Nacional de las Personas (RENAPER) y dispuso la creación de la Libreta Cívica. Este nuevo sistema fue, de acuerdo al autor, el que facilitó a los indígenas la obtención de la documentación.

24 Este autor destaca que la tonelada con que se pagaba al indígena en el Chaco pesaba 700 kilos (Bialet Massé, 1904, pp. 41). En Europa, se presentó el mismo problema con el trabajo a destajo. Por ejemplo, en la
El desconocimiento y la desconfianza del indígena sobre el funcionamiento de la justicia también emerge en forma clara en el informe de este autor. En el capítulo II, relata las siguientes circunstancias que le tocó presenciar en el Chaco.

Un indio del Chaco oriental conserva en su poder una multitud de contratos. No sabe leer ni escribir; pero uno está doblado en cuatro, otro a lo largo, otro en punta, y otro señalado con una línea roja y otro con una negra, y así los distingue todos. Ninguno le ha sido cumplido (Bialet Massé, 1904, pp. 34).

En las nuevas circunstancias, ya no existía la posibilidad de contar con cautivos y seguramente serían pocos los refugiados a los que se pudiera acudir para tener certeza de lo que estaba escrito. En este marco, el siguiente comentario muestra una nueva práctica de traducción unida al silencio.

Es curioso observar al indio con su papel, cuando cree quele han faltado al contrato; busca un cristiano yle dice: «Cristiano, lee». Después refiere lo que le han hecho, se calla, se va. No protesta, pero no lo olvida nunca. Cuando llega la ocasión saca sus papeles doblados del tubo y hace la cuenta del último centavo que le robaron (Bialet Massé, 1904,p. 35).

La ausencia de toda denuncia o investigación de oficio y de búsqueda de testimonios entre el episodio de Rincón de la Bomba en octubre de 1947 y el ańo 2005 constituyó, a nuestro juicio, un resultado claro del contexto de Silencio del Indígena. El contexto de "Trato Equitativo"

minería de carbón, el pago recibido por el trabajador dependía del peso del producto extraído por el mismo, sobre el cual existían conflictos permanentes. En Inglaterra, en 1872, una ley otorgó el derecho a los trabajadores a nombrar un "verificador de pesos", pagado por los propios mineros para controlar esta operación. Ver Dobb (1941). 
La igualdad frente a la ley es, de acuerdo a Bengoa (1995), uno de los aspectos que impulsó el movimiento que denomina "indigenismo". Tuvo como uno de sus primeros antecedentes la participación protagónica de indígenas en la Revolución de Méjico de 1910. Inicialmente, en este movimiento, adquirieron un papel predominante no los propios indígenas, sino humanistas y antropólogos, entre otros. Formalmente, su inicio puede localizarse en el Congreso Indigenista Interamericano, celebrado en la ciudad de Patzcuaro, México, en 1941 (Lenton, 1999; D 'Addario, 2013).

En este marco internacional, comenzó gradualmente la aplicación de políticas indigenistas a nivel estatal y continental en pos de la igualdad de derechos. Existió, además, una mayor preocupación por parte de los Estados por el cumplimiento efectivo de los derechos civiles de las comunidades indígenas, aun cuando prevalecía una visión orientada hacia la asimilación de la cultura aborigen.

El indigenismo fue un movimiento de defensa de los derechos civiles existentes, el cual intentaba limitar la discriminación negativa, de naturaleza similar a la que existió en USA en relación a los habitantes afroamericanos. No obstante, este movimiento no se planteaba la creación de nuevos derechos, particulares de las culturas indígenas, $\mathrm{ni}$ mucho menos, la plurinacionalidad. Solo el período previo de sometimiento y silencio del indígena justifica la existencia posterior del indigenismo.

En materia laboral, cabe señalar el Convenio 107 de la OIT, aprobado por la Argentina en 1959 mediante la Ley 14.932. Esta norma, que equiparaba derechos, no fue, sin embargo, incorporada efectivamente a la normativa en materia laboral hasta la sanción de la Ley 23.302 en el año 1985 (Lenton, 2010).

Un elemento importante a destacar es la organización que fueron adquiriendo gradualmente las comunidades indígenas y el cambio en las políticas públicas hacia las mismas, que variaban desde el énfasis en la "protección" hasta la colaboración y el empoderamiento. Se creó, en 1958, la Dirección Nacional de Asuntos Indígenas y, posteriormente, en 1985, el Instituto Nacional de Asuntos Indígenas (INAI). La ley 23.302 del año 1985 facilitó a las propias comunidades presentarse ante la justicia no como individuos aislados, sino como entidades con personería jurídica propia y un mejor asesoramiento legal ${ }^{25}$.

Finalmente, los Tratados Internacionales en materia de Derechos Humanos, a los cuales adhirió la Argentina y que adquirieron rango legal a partir de la reforma constitucional de 1994, terminaron por instituir internamente el principio de igualdad ante la ley.

El contexto de Trato Equitativo incluye otros elementos que, desde un punto de vista formal, pueden considerarse como una suerte de discriminación positiva en materia de derechos, pero que sin los mismos la igualdad frente a la ley puede resultar una ilusión. Para aquellas comunidades cuya forma de propiedad de la tierra debe ser comunitaria, la inexistencia de este derecho lo coloca en una situación de clara vulnerabilidad. Otro tanto ocurre con la necesidad de contar, en algunos casos, con traductores de la lengua originaria.

El proceso que se inicia con la denuncia del año 2005 implica una situación propia del contexto de Trato Equitativo, aunquela lentitud del accionar de la justicia y la forma en que se reciben los testimonios serían cuestionables.

25 Para un análisis de los inicios de este proceso y el papel protagónico que van adquiriendo los indígenas, ver Lenton (2010). El reconocimiento jurídico de las Comunidades Indígenas se acepta ya en la Ley 23.302 del año 1985. 


\section{Conclusiones}

En este trabajo, se ha propuesto analizar cómo se ha desarrollado a lo largo del tiempo, luego de constituirse la Argentina como un Estado-Nación, la relación con los miembros de comunidades indígenas, vencidos militarmente a fines del siglo XIX en la Patagonia y a principios del siglo XX en Chaco.

Dado lo amplio de la temática, se ha hecho foco en un aspecto específico de ella: los casos de conflictos interétnicos tratados por el Poder Judicial y, dentro de los mismos, los procesos en los que se ha escuchado el testimonio de los indígenas, particularmente cuando los mismos fueron contrarios a lo manifestado por funcionarios o empresarios socialmente importantes.

La investigación se ha diseñado como estudio de dos casos particularmente graves por los derechos que se vulneraron y la cantidad de indígenas afectados. Se han descripto los mismos a partir de los antecedentes con que se cuenta y se han propuesto explicaciones.

El primer caso es un Contrato de Locación de Servicios del año 1899, por el cual se contrataron mujeres de la etnia pilagá para ser exhibidas en la Feria Internacional de Paris del año 1900. El segundo es la última masacre de indígenas de esta misma etnia, ocurrida en el paraje Rincón de la Bomba en Formosa, en el año 1947.

En ambos casos, intervienen previamente funcionarios, cuya misión era velar por la protección de los indígenas: en el primer caso, el Defensor de Menores e Incapaces y, en el segundo, la Dirección de Protección al Indígena. Ninguna de estas intervenciones impidió que se violaran derechos básicos: la libertad y la vida, respectivamente.

Con respecto al Contrato de Locación de Servicios, se ha desarrollado la explicación del marco jurídico en que se encontraban los indígenas en el año 1900, particularmente su encuadre como incapaces jurídicos, y del rol dado en este marco a los defensores de menores e incapaces para que los representen. Si bien el testimonio de las mujeres indígenas finalmente fue escuchado, lo excepcional del caso indica que se estaba muy lejos de un principio siquiera formal de igualdad frente a la ley.

Estos testimonios fueron solicitados y escuchados recién en Buenos Aires, sitio muy lejano al lugar de origen de las mujeres pilagá. Pero, además, esto no era necesario de acuerdo al status jurídico que tenían los indígenas, sino que se realizó por razones políticas, puesto que el caso había llamado la atención de la opinión pública porteña, lo que derivó en el interés del gobierno nacional y en la intervención de la Corte Suprema de Justicia de la Nación. Solo estas consideraciones políticas pueden explicar que finalmente se le haya otorgado valor al testimonio de las indígenas, consideradas hasta ese momento como jurídicamente incapaces.

En el segundo caso, el testimonio de los indígenas solo fue escuchado mucho tiempo después, transcurridos casi 60 años del hecho y cuando la mayoría de los responsables ya había fallecido.

Para encuadrar este último caso, hemos propuesto una explicación histórica más amplia, basada en "contextos", cuyos cambios dan lugar a que finalmente se escuchasen los testimonios. Hemos sugerido tres contextos que, si bien no son determinantes en cada conflicto que involucra a comunidades indígenas en particular, sí deben permitir inferir cuál ha sido la modalidad predominante de tratamiento de los conflictos. Estos contextos incluyen aspectos normativos y prácticas políticas y sociales, y los hemos denominado de "Seguridad-Peligrosidad", de "Silencio del Indígena" y de "Tratamiento Equitativo". 
La masacre habría ocurrido dentro del primer contexto, el segundo contexto explica el largo período transcurrido sin que medie denuncia ni investigación de oficio, y el tercero esclarece que, finalmente, se hayan llevado a cabo la denuncia y la toma de testimonios de indígenas.

Si bien los casos analizados se consideran particularmente importantes por su gravedad, una mejor comprensión de la problemática podría lograrse con una investigación de campo de mayor alcance. $\mathrm{Si}$ se indagara a los propios miembros de las comunidades indígenas y se estudiaran los archivos judiciales de diversas jurisdicciones en la Argentina, se podrían obtener mayores evidencias sobre los momentos en que comenzaron a ser tratados, en el ámbito judicial, los conflictos entre integrantes de comunidades indígenas y otros grupos sociales. Una investigación de este tipo permitiría precisar respuestas a preguntas tales como: ¿cuándo se empezaron a radicar denuncias?, ¿qué papel jugaron las instituciones que debían representarlos o protegerlos?, ¿cuándo se dio inicio a la intervención de organizaciones indígenas con personería jurídica?, ¿̨cuándo comenzó a incorporarse el testimonio de los indígenas como prueba?, ¿a partir de qué momento se procuró contar con traductores para aquellos casos en que fuese necesario?, ¿qué trato se dio a aquellos casos en que los testimonios de indígenas eran contrarios a los testimonios de otros miembros de la sociedad?, entre otras preguntas posibles.

Este proceso no necesariamente haocurrido en forma simultánea en todos los ámbitosgeográficos del país ni en referencia a los distintos tipos de derechos afectados. Cuando se observa el episodio de Rincón de la Bomba pareceque, en algunos casos, no ha pasado mucho tiempo desde que la igualdad efectiva ante la ley ha comenzado a implementarse.

\section{Bibliografía}

- Andre, M. L. (2012, 28 de julio). Matanza de Napalpi [Archivo de video]. Recuperado de https://www.youtube.com/ watch?v=2a3kSDKfi48

— Bechis, M. (2006). "La 'Organización Nacional' y las Tribus Pampeanas en argentina durante el siglo XIX". Revista TEFROS, 4(2), pp. 1-24.

— Bengoa, J. (1995). "Los indígenas y el Estado Nacional en América Latina”. Revista de Antropología, 38(2), pp. 151-186.

- Bialet Massé, J. (1904). Informe sobre el estado de las clases obreras en el interior de la República. Vol. 1. Buenos Aires: Editorial Adolfo Grass.

— Borrero, J. M. (1989). La Patagonia trágica. Buenos Aires: Zagier \& Urruty.

—Carrasco, M. (2016). "Entre nosotros: cultura y pericias antropológicas en la justicia penal”. Antropologías del Sur, 3(6), pp. 65-87.

—Cunill, C. (2011). "El indio miserable: nacimiento de la teoría legal en la América colonial del siglo XVI". Cuadernos Intercambio sobre Centroamérica y el Caribe, 8(9), pp. 229-248.

—D' Addario, L. M. (2013). “Las políticas Estatales hacia los Pueblos indígenas y las prácticas de normalización durante el primer peronismo Perspectivas de análisis desde un es". En XIV Jornadas Interescuelas. Departamento de Historia de la Facultad de Filosofía y Letras, Universidad Nacional de Cuyo. Recuperado de http://cdsa. aacademica.org/000-010/513.pdf

- de Souza Santos, B. y Exeni Rodríguez, J. L. (Eds.) (2012). Justicia Indígena, plurinacionalidad e interculturalidad en Bolivia. La Paz: Fundación Rosa Luxemburgo/ Abya-Yala

—Delrio, W. (2002). "Indios amigos, salvajes o argentinos. procesos de construcción de 
categorías sociales en la incorporación de los pueblos originarios al Estado-Nación (1870-1885)”. En L. Nacuzzi (Comp.), Funcionarios, diplomáticos, guerreros. Miradas hacia el otro en las fronteras de Pampa y Patagonia (siglos XVIII y XIX) (pp. 203-245). Buenos Aires: Sociedad Argentina de Antropología.

- Delrio, W. (2010). Memorias de expropiación Sometimiento e incorporación indígena en la Patagonia (1872-1943). Bernal: Universidad Nacional de Quilmes Editorial.

-Delrio, W., Lenton, D., Musante, M., Nagy, M., Papazian, A. y Pérez, P. (2010). "Del silencio al ruido en la historia. Practicas genocidas y pueblos originarios en Argentina”. En Actas III Seminario Internacional Políticas de la Memoria. Centro Cultural de la Memoria Haroldo Conti. Recuperado de http://conti.derhuman.jus.gov.ar/2010/10/ mesa-36/delrio_lenton_musante_nagy_papazian_perez_mesa_36.pdf

—Dirección de Servicios Legislativos. (2018). Pueblos Originarios Legislación e Informes Internacionales Legislación nacional y provincial Doctrina y Jurisprudencia. Buenos Aires: Biblioteca del Congreso de la Nación. - Dobb, M. (1941). Salarios. México: Fondo de Cultura Económica.

- Escolar, D. (2008). "El repartimiento de prisioneros indígenas en Mendoza y la teorización nativa del 'criollo', décadas de 1880-1940”. En Actas III Jornadas de Historia de la Patagonia. Recuperado de http://www.hechohistorico.com.ar/Trabajos/Jornadas\%20de\%20Bariloche $\% 20$ -\%202008/Escolar.pdf

- Fandos, C. A. y Teruel, A. A. (2012). “Cómo quitarles esas tierras en un día después de 200 años de posesión? Enfiteusis, legislación y práctica en la Quebrada de Humahuaca (Argentina). Bulletin de l'Institut français d'études andines, 41(2), pp. 209-239.

— Foucault, M. (2007). La verdad y las formas jurídicas. Buenos Aires: Editorial Gedisa.

-Lanata, J. L. (Comp.) (2014). Prácticas Genocidasy Violencia Estatal en Perspectiva Transdisciplinar. San Carlos de Bariloche: IIDyPCa-CONICET. Recuperado dehttps:// iidypca.homestead.com/Pr_cticas_Genocidas_y_Violencia_Estatal_en_perspectiva_transdisciplinar..pdf

— https://iidypca.homestead.com/Pr_cticas_Genocidas_y_Violencia_Estatal_en_ perspectivatransdisciplinar..pdfLenton, D. (1997). "Los Indígenas y el Congreso de la Nación Argentina: 1860 -1976”. Revista NAyA, 2(14).

— Lenton, D. (1999). "Los dilemas de la ciudadanía y los indios-argentinos: 18801950". Publicar: en Antropología y Ciencias Sociales, (8), pp. 7-30.

- Lenton, D. (2010). "Políticas del Estado Indigenista y políticas de representación indígena: propuestas de análisis en torno al caso neuquino en tiempos del desarrollismo. Sociedades de paisajes áridos y semiáridos, 2(2), pp. 85-108.

— Lenton, D. (2014). "Apuntes en torno a la aplicabilidad del concepto de genocidio en la historia de las relaciones entre el estado argentino y los pueblos originarios”. En J. L. Lanata (Comp.), Prácticas Genocidas y Violencia Estatal en Perspectiva Transdisciplinar (pp. 32-51). San Carlos de Bariloche: IIDyPCa-CONICET. Recuperado dehttps:// iidypca.homestead.com/Pr_cticas_Genocidas_y_Violencia_Estatal_en_perspectiva_transdisciplinar..pdf

—Lenton, D. (2017). "El expediente de la sublevación de los pampas en Santa Ana 1 (1888, Misiones, Argentina)". Corpus: archivos virtuales de la alteridad americana, 7(1), pp. 1-44.

—Levaggi, A. (1990). "La protección delos naturales por el Estado argentino (1810-1950): el problema de la capacidad". Revista Chilena de Historia del Derecho, (16), pp. 445-469. 
— Levaggi, A. (1992). Muerte y resurrección del derecho indiano sobre el aborigen en la Argentina del siglo XIX. Jahrbuch für Geschichte Lateinamerikas / Anuario de Historia de America Latina, (29)1, pp. 179-194.

- Mapelman, V.y Varreire, G. (Productoras) y Mapelman, V. (Directora). (2010). Octubre pilagá: relatos sobre el silencio. Argentina: DVCAM. Recuperado de https:// www.youtube.com/watch?v=_-RrW15G2kI —Mariategui, J. C. [1928] (1996). Siete ensayos de interpretación de la realidad peruana. Lima: Empresa Editora Amauta S.A.

- Martínez Sarasola, C. M. (1998). Los hijos de la tierra: historia de los indígenas argentinos. Buenos Aires: EMECE Editores. - Matarrese, M. (2017). "Qom Pi: un territorio atravesado por la violencia entre lo propio y lo ajeno". Publicar: en Antropología y Ciencias Sociales, (22), pp. 25-44. - Matarrese, M. (2018). "Política indigenista y política indígena pilagá en materia de tenencia de la tierra. Question, 1(58), pp. 1-17.

- Memorias de la tierra. (2012, 1 de junio). Masacre en Rincón de la Bomba: gendarmes a indagatoria [Archivo de video]. Recuperado de https://www.youtube.com/ watch?v=P0W4AxUePv8.

— Nagy, M. y Papazian, A. (2011). "El campo de concentración de Martín García. Entre el control estatal dentro de la isla y las prácticas de distribución de indígenas (1871-1886)" Corpus: archivos virtuales de alteridad americana, 1(2), pp. 1-35.

- Perez, P. (2015). "Futuros y fuentes: las listas de indígenas presos en el campo de concentración de Valcheta, Río Negro (1887)". Nuevo Mundo Mundos Nuevos. Recuperado de https://journals.openedition. org/nuevomundo/68751?lang=en

—Pigna, F. (2004). Los mitos de la historia argentina 2. Buenos Aires: Editorial Planeta. —Peró, J., Brea, R., Moncayo, G. y Alonso
Gómez, A. (1969). Fallos de la Corte Suprema de Justicia de la Nación. Vol. 274. Banfield: Corte Suprema de Justicia de la Nación.

— Ranzani, O. (2010, 12 de abril). "Una masacre que debía ser contada. Entrevista a Valeria Mapelman, directora de Octubre Pilagá, relatos sobre el silencio". Página 12. Recuperado de https://www. pagina12.com.ar/diario/suplementos/ espectaculos/2-17576-2010-04-12.html 